\title{
Bach's symphony for metastasis
}

As tumours progress, the metabolic output of cancer cells is elevated, culminating in increased production of reactive oxygen species (ROS). To counterbalance oxidative stress and ensure redox homeostasis, a subgroup of non-small-cell lung cancers acquire stabilizing mutations in NFE2L2, the gene encoding NRF2, the transcriptional regulator of the cellular antioxidant response, and inactivating mutations in its negative regulator, KEAP1. The transcriptional regulator $\mathrm{BACH} 1$ negatively controls the expression of antioxidant genes, counteracting NRF2 activity. Now, two studies provide complementary evidence demonstrating that both endogenous and exogenous antioxidants can induce stabilization of $\mathrm{BACH} 1$ to promote lung cancer metastasis.

Lignitto et al. began their study by investigating whether KEAP1 inactivation affected metastasis in a Kras $^{\mathrm{LLL}-\mathrm{G} 12 \mathrm{D} /+} ; \operatorname{Trp} 53^{\mathrm{f} / \mathrm{fl}}(\mathrm{KP})$ mouse model of lung adenocarcinoma. Knockout of Keap1 on the KP background (KPK mice) resulted in more thoracic and lymph node metastases relative to KP mice. RNA sequencing of cell lines generated from KP and KPK lung tumours revealed upregulation of metastatic genes in Keap1 mutant cells.

Searching for a transcriptional driver that might be responsible for the gene enrichment led to the identification of NRF2 and BACH1.

$\mathrm{BACH} 1$ is known to be degraded by the proteasome in response to high levels of intracellular free haem, a potent pro-oxidant molecule that can be generated by ROS. NRF2 accumulation upon oxidative stress can activate the transcription of haem oxygenase 1 (HO1), which catabolizes haem. Interestingly, Lignitto et al. noted that $\mathrm{BACH} 1$ protein levels were increased in KPK cells compared with KP cells and that disruption of the NRF2-KEAP1 interaction with a small molecule inhibitor in $\mathrm{KP}$ cells could cause BACH1 protein to accumulate. Together, these data suggested that increased
NRF2 levels upon KEAP1 loss could promote BACH1 stabilization. Moreover, under these conditions in which NRF2 activation is upregulated, $\mathrm{HO} 1$ protein levels were also higher, intimating that $\mathrm{BACH} 1$ stabilization is likely the result of increased HO1-mediated catabolism of haem. Further experiments revealed that the ubiquitin ligase CRL1-FBXO22 targets $\mathrm{BACH} 1$ for degradation in the presence of haem.

To examine how exogenous antioxidants might influence lung cancer metastasis, Wiel et al. also made use of the $\mathrm{K}$ and $\mathrm{KP}$ mouse models. They found that supplementing the diet of lung tumour-bearing mice with either a pharmacological or dietary antioxidant, $N$-acetylcysteine (NAC) and vitamin $\mathrm{E}$ respectively, was sufficient to increase the amount of lymph node metastases relative to untreated control mice. Of note, primary tumour growth as well as survival of the mice were unaffected by either treatment.

Cell lines derived from tumours of control and NAC-treated $\mathrm{K}$ mice (con-K and NAC-K hereafter) were then used to gain a mechanistic understanding of the pro-metastatic antioxidant effects. The NAC-K cells had an increased ability to migrate and invade compared with con-K cells, which was concomitant with an enrichment of genes involved in cell motility and migration.

Consistent with a reduction in ROS levels, NAC-K cells also exhibited downregulation of genes associated with antioxidant processes, in particular the target genes of NRF2 and $\mathrm{BACH} 1$.

Similar to Lignitto et al., these authors noted that BACH1 protein levels were higher in NAC-K cells than con-K cells and that this could be reversed by the addition of haemin, a haem derivative. This suggested that antioxidants, by reducing ROS levels, prevent the release of free haem and, in doing so, the degradation of BACH1.

Both studies then confirmed that $\mathrm{BACH} 1$ stabilization could drive metastasis by showing that subcutaneous implantation of Bach1depleted KP or KPK cells decreased lung metastases and that intravenous injection of Bach1-deficient NAC-K cells inhibited metastasis to the lung. Furthermore, pharmacological inhibition of HO1 with ZnPPIX was able to reduce the metastatic potential of both subcutaneous $\mathrm{KP}$ and KPK tumours by inducing $\mathrm{BACH} 1$ destabilization in a FBXO22mediated manner, indicating a possible therapeutic avenue.

When addressing which set of BACH1 target genes accounted for the elevated metastasis, Wiel et al. identified a network of genes ascribed to metabolic processes and in particular to glycolysis (such as hexokinase 2 (Hk2) and Gapdh). This was consistent with the $50 \%$ increase in the rate of glycolysis of NAC-K cells compared with con-K cells, which was dependent on BACH1 expression. Moreover, targeting glycolysis in vivo through inhibition of GAPDH with 3-bromopyruvate could block the antioxidant-induced metastasis of NAC-K cells.

Last, examination of human lung adenocarcinoma samples by Lignitto et al. revealed that both $\mathrm{BACH} 1$ and $\mathrm{HO} 1$ expression were increased in metastases relative to matched primary tumours and that a $\mathrm{BACH} 1$ signature correlated with poor survival.

Therefore, BACH1 stabilization can occur both in the presence of high or low NRF2 activity through the regulation of haem levels. Collectively, the two studies offer insight into the complex and context-dependent role of ROS in tumour progression.

Anna Dart

ORIGINAL ARTICLES Lignitto, L. et al. Nrf2 activation promotes lung cancer metastasis by inhibiting the degradation of Bach1. Cell 178 316-329.e18 (2019) | Wiel, C. et al. BACH1 stabilization by antioxidants stimulates lung cancer metastasis. Cell 178, 330-345.e22 (2019) 